\title{
A framework for manipulation and locomotion with realtime footstep replanning
}

\author{
Duong Dang, Florent Lamiraux and Jean-Paul Laumond
}

\begin{abstract}
This paper focuses on realization of tasks with locomotion on humanoid robots. Locomotion and whole body movement are resolved as one unique problem. The same planner and controller are used for both stages of the movement. Final posture and footprint placements are found by resolving an optimization problem on the robot augmented by its footprints. Footstep replanning is done in realtime to correct perception and execution errors. The framework is demonstrated with the HRP-2 robot in a number of different scenarios.

Index Terms-locomotion, footsteps, replanning, realtime, visual servoing
\end{abstract}

\section{INTRODUCTION}

$\mathbf{L}$ OCOMOTION and manipulation are among the most exciting topics in humanoid robotics research. Numerous works have been carried out in the past years in walking and running generation [1]-[7], notably with the introduction of the zero momentum point (ZMP). On manipulation, task-based methods have been developed since the eighties of the last century for industrial robot and robotic arms, [8], [9]. These methods have been extended to humanoids in recent years as more and more robots have been made available for research [10]-[12].

While both locomotion and manipulation generate on their own great interests for research and can be treated separatedly as is often the case, it is intriguing how much one can extend the capability of robots when combining these two problems in the same context. With locomotion coupled with manipulation, the robot is no longer constrained to manipulate only objects in its workspace. In fact, the workspace is extended with locomotion that just becomes another task. In addition to creating new capability and new task, there is a real need to build the bridge between locomotion and manipulation. There are many cases where the coupling is so important that one cannot separate both problems. A humanoid robot might need to make steps before manipulating some objects. A dynamically demanding manipulation scenario might require that the robot end up at some exact configuration at the end of the locomotion stage. For instance, to manipulate an object at ground level, constraints have to be put on feet placement and affect the way locomotion should be conducted.

Resolving locomotion and a complex upper body manipulation is, however, not a simple task in practice. Typically, a computationally expensive planning stage is involved at first. The resulting plan may be expressed either in configuration

This work was not supported by any organization

D. Dang, J-P. Laumond and F. Lamiraux are with CNRS, LAAS, 7, avenue du Colonel Roche, F-31077 Toulouse, France. nddang@laas. fr

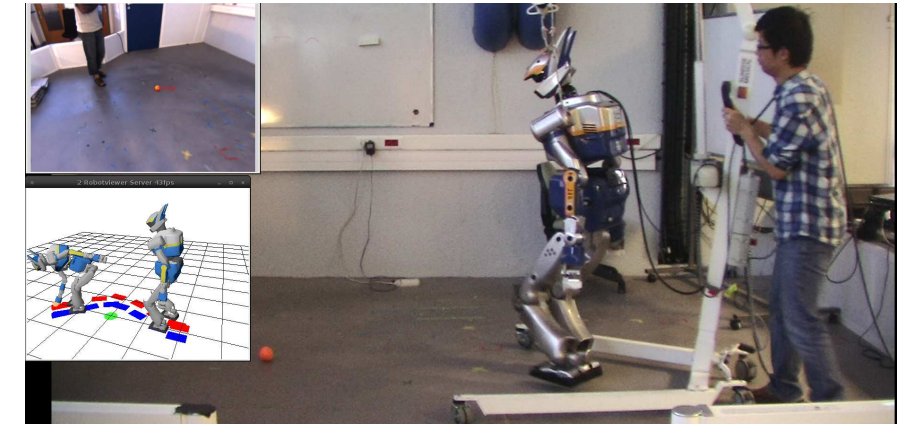

Fig. 1. Footprints replanning during locomotion in a waling-grasping scenario on HRP2. (Videos available at http://homepages.laas.fr/nddang/humanoids2011)

space, which can be executed directly on the robot, or in terms of tasks to be given to the controller. Yet, no sensing system is perfect, the information obtained at the planning stage is usually different from reality. In addition, execution errors, due for instance to sliding while walking make open loop execution unrealistic. As a consequence, correction has to be done online. This is a challenging task since a time-consuming replanning can prevent the robot from being reactive. Online generation of footsteps have been studied previously by several research groups [13]-[15] but usually in the context of a decoupled locomotion problem.

In this paper, a framework is proposed to treat both manipulation and locomotion with online replanning. Sources of errors such as perception system, execution error, moving objective are taken into account online by the motion planner, which in turn, produces in real time up-to-date instructions to the controller in the form of task space trajectories for the feet (steps) and for the upper-body (manipulation).

\section{Approach and contribution}

The motion planner in this paper extends the work of Kanoun et al. [16], [17], where robot motion is resolved in the form of an optimization problem. The humanoid robot is augmented by its footprints and tasks are expressed on this this augmented robot. Modifications have been made to the initial method in order to make it run on-line. First, instead of resolving the problem in the configuration space of the robot, only an intermediate state of the resolution is performed and fed to the controller: namely final posture and foot placements during walking.

The main contribution of this paper is the integration of the method described in [16] into a control framework that 


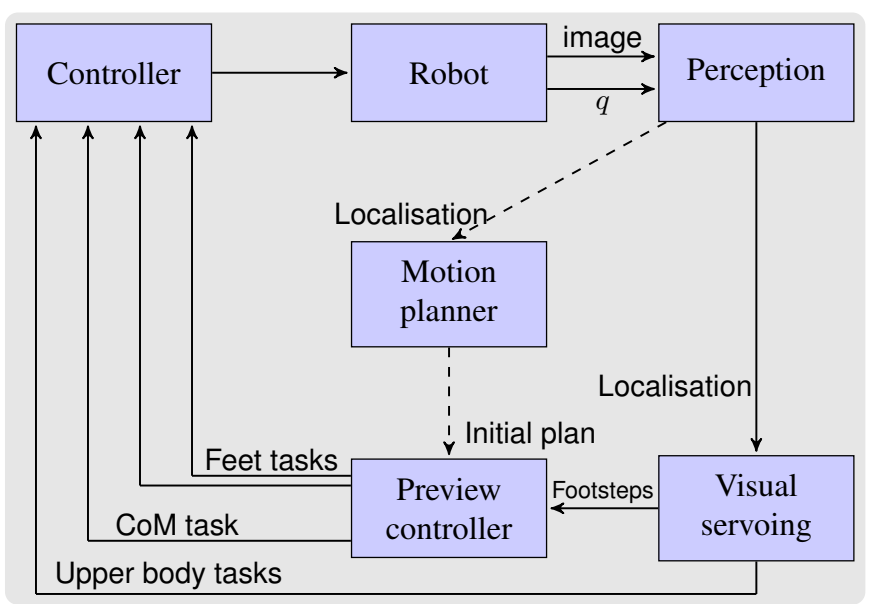

Fig. 2. Architecture

achieves real time replanning during walking. The entire framework is then validated with a number of different scenarios on the dynamic simulator OpenHRP and validated on the real robot with the scenario described in Section IV-C (Figure 1).

\section{IMPLEMENTATION}

\section{A. Architecture overview}

Figure 2 depicts how different components interact with each other. At first, a complete plan is made and fed to the system. This is done only once hence the dotted arrow. In the execution loop, images from the robot cameras are transmitted to the perception module. This module analyses the images to detect and localize objects of interest in the environment with respect to the robot. The visual servo uses 3D position of tracked object to replan in real time upcoming footprints and upper body tasks (hand task, posture task, etc.). Footprints are passed to a preview controller which also computes trajectories of critical operation points of the lower body, which is responsible for walking or keep the robot balanced while standing, namely, trajectories of left foot, right foot and the center of mass. The controller combines these tasks all together within a prioritized stack and computes the appropriate control signal that is sent to the robot. Since the controller makes no distinction between walking and object manipulation, it only executes tasks, there is no "gap" between the walking and manipulation stages.

\section{B. Footstep and posture planning}

The core of the plannner is the resolution of a hierachical system of inequality and equality tasks [16], [17]. The special feature of this approach is the possibility of having inequality tasks in higher priority than equality task. This is extremely helpful in humanoid robots where a lot of contraints have to be expressed as inequality tasks.

1) Prioritized linear and inequality systems: Considering a system of linear equalities and inequalities:

$$
\begin{aligned}
& A x=b \\
& C x \leq d
\end{aligned}
$$

for which the solution can be expressed as an optimization problem

$$
\min _{x \in \Omega, w \in \Re^{n}} \frac{1}{2}\|A x-b\|^{2}+\frac{1}{2}\|w\|^{2}
$$

subject to

$$
C x-w \leq d
$$

In an prioritized linear system, one can resolve 3 iteratively at each level as follows:

$$
\begin{aligned}
S_{0} & =\Re^{n} \\
S_{k+1} & =\arg \min \min _{x \in \Omega, w \in \mathcal{R}^{n}} \frac{1}{2}\|A x-b\|^{2}+\frac{1}{2}\|w\|^{2} \\
& \text { subject to } C_{k} x-w \leq d_{k}
\end{aligned}
$$

In robotics, a task is typically represented by its Jacobian $J$. The classic example is a position task in operational space with $T(q)=0$ is the goal and $T(q)=c$ the current error. The solution $\dot{q}$ to achieve exponential convergence $c=C_{0} \exp (-\lambda t)$ is written as

$$
J \dot{q}=-\lambda c, J=\frac{\partial T}{\partial q}(q)
$$

Other tasks can be written as inequalities $T(q) \leq 0$ such as collision and self-collision avoidance, joint limits, etc: The resolution of a prioritized set of task can be computed by:

Find $\dot{q}^{*} \in S_{k}$ :

$$
\begin{aligned}
& S_{0}=\Re^{n} \\
& S_{i}=\arg \left\{\min _{\dot{q} \in S_{i-1}} \frac{1}{2}\left\|J_{i} \dot{q}-e_{i}\right\|^{2}\right\} \text { for equality tasks } \\
& S_{i}=\arg \left\{\min _{w, \dot{q} \in S_{i-1}} \frac{1}{2}\|w\|^{2} \quad \text { s.t. } \quad J_{i} \dot{q}-e_{i} \leq w\right\}
\end{aligned}
$$

for inequality tasks

2) Application to footstep planning: As explained earlier, the determination of footsteps and final posture defining the motion executed on the robot is achieved in one problem by using the previous resolution of equality and inequality system. The main idea of this approach is to add a virtual joint (Figure 3) with three degree of freedoms for each step. Let us consider a robot of $n$ degrees of freedom executing $k$ steps, the resulting virtual kinematic chain adds $3 k$ degrees of freedom to the real robot to form a $n+3 k$ d.o.f. kinematic chain. Constraints on how far the robot can physically step or turn become joint limits for theses new joints. The constraints that the robot should not step one foot on one another becomes auto-collision avoidance. On this new robot with this set of constraints in addition, a set of appropriate tasks can then be applied, namely: 


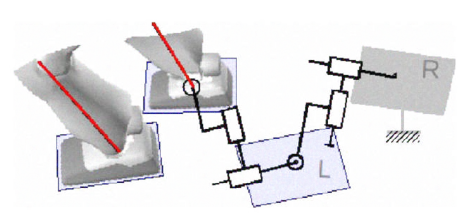

Fig. 3. Virtual kinematic chain

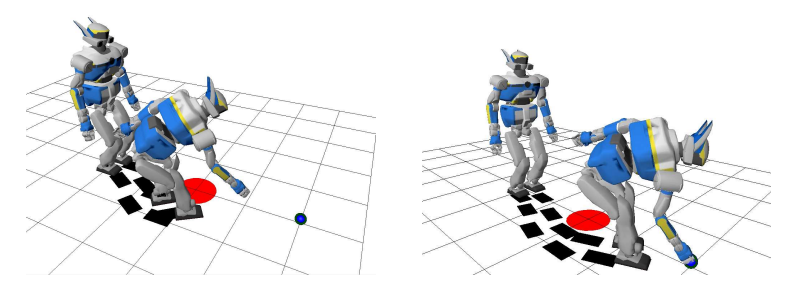

Fig. 4. Deployment of virtual kinematic chain

- inequality constraints, in order, joint limits, projection of the center of mass, self-collision avoidance of robot, selfcollision avoidance for the virtual manipulator, position and orientation of the supporting foot

- object manipulation task. e.g. grasp, reach for an object with 2 hands, etc.

- parallel task for the upper body during walking,

- gaze task (keep objects of interest in the vision field).

Figure 5 depicts how the upperbody task "attracts" the augmented robot hence initiate footeps. The last $n$ degrees of freedoms in the result represent the final posture, and the final position of the virtual kinematic chain represents footsteps. The complete motion in configuration space can then be found by passing the footsteps to a preview controller which output trajectories for left foot, right foot and the center of mass. These trajectories in turn, in addition with the upper body task at the final step can be given as equality tasks to the prioritized stack. The resulting motion is therefore never computed before-hand. It is instead the result of the control architecture.

Determine the number of steps: In the previous section, it was assumed that the number of steps $k$ was known before hand. However, the target detected by the vision system can be found in a wide range of distance. Therefore, it is difficult to guess in advance how many steps the robot should make. One important point in this section and also the first modification made on this paper to the original method is the fact this parameter $k$ can be found automatically by the optimizer. The algorithm is summarized as follows:

Figure 5. depicts the exploration of the virtual kinematic chain in space.

\section{ONLINE FOOTSTEP REPLANNING}

One big challenge in our context is the use of stereo-vision to detect and localize objects. Localization errors grow dramatically with the distance between the object and the sensor. Usually, this distance is maximal in the initial configuration of the robot, when motion planning is performed.
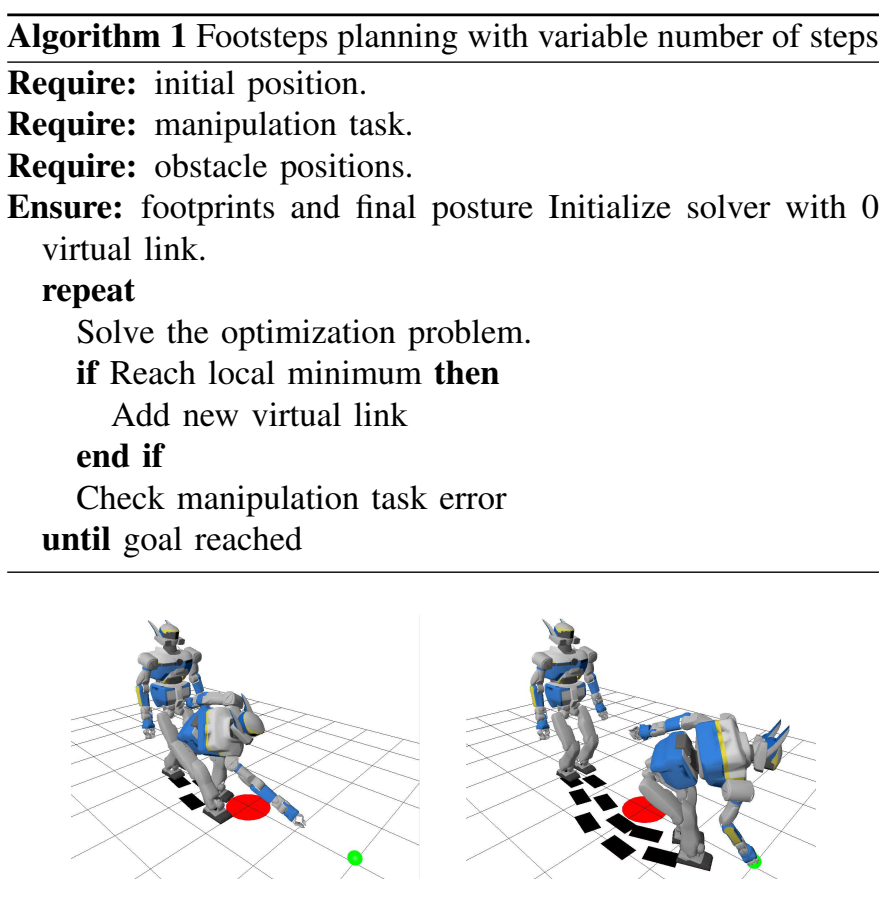

Fig. 5. Find the number of steps automatically by the optimizer

The resolution to a complete motion in joint space described in previous section typically takes up to one minute for a long motion. Yet, to be reactive, replanning should finish at least once every stepping interval of the robot (typically under a second). It is clear that replanning the motion up to configuration space motion is not feasible in practice.

\section{A. Footsteps and final posture replanning}

As explained ealier intermediate results, i.e. final posture and footsteps, of the planning stage are sufficient for the controller. This is where replanning becomes feasible since the computation of footsteps and posture is typically from 3 to $10 s$, a replanning could be well below $1 s$ and hence guarantee reactivity. The augmented robot then starts at previous state and update its tasks according to new sensory information about landmarks. Algorithm 2 describes the stages of planning, the results are shown in Figure 6. The solver converges more quickly than planning footsteps and posture from initial position since the current configuration is already closed to the goal configuration. Table I shows replanning time for a grasping task with goal as a ball on the ground at a distance around 2 meters. The modification is taken randomly in arbitrary directions.

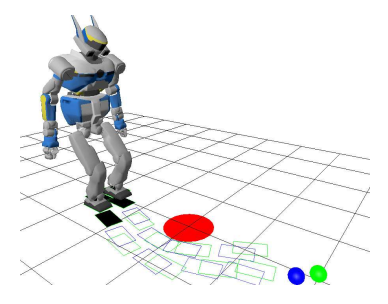

Fig. 6. Footsteps replanning 


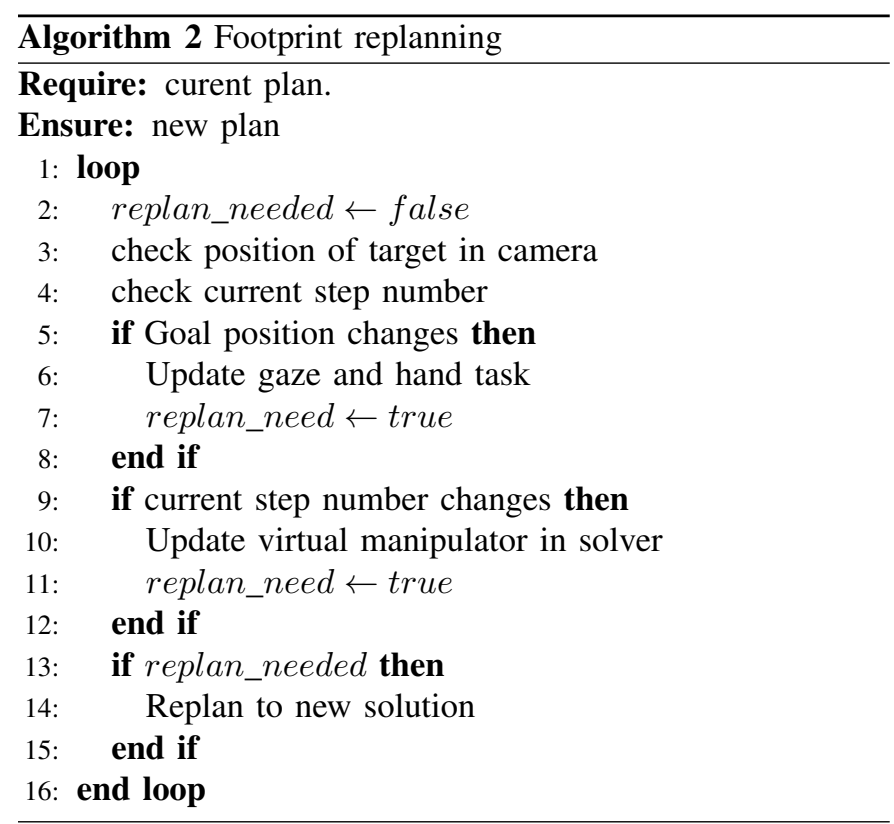

\begin{tabular}{c|r|r|r} 
Goal modification $(\mathrm{m})$ & Max $(\mathrm{ms})$ & Min $(\mathrm{ms})$ & Average $(\mathrm{ms})$ \\
\hline 0.01 & 2615 & 251 & 595.1 \\
0.02 & 2159 & 275 & 673.2 \\
0.05 & 2343 & 488 & 920.7 \\
0.1 & 2498 & 593 & 1299.8 \\
0.2 & 4166 & 608 & 1977.0 \\
0.5 & 7123 & 610 & 3685.3
\end{tabular}

TABLE I

CPU TIME FOR REPLANNING OF FINAL POSTURE AND FOOTSTEPS (MILISECONDS) FOR A LOCOMOTION-GRASP TASK (FIGURE 1)

The replanning process for the whole-body and footsteps depends greatly on configuration and it is not guarantied a computing time less than stepping time $(0.8 s)$. Without modification, small corrections (e.g. due to drifting) can be dealt with. Otherwise, an execution strategy must be applied at the planning-control bridge to deal with large errors, such as the case of stereo vision system.

Stop and go: One obvious approach to address the realtime issue is to stop the robot or let it step in place if the planner takes too long to response.

Interative optimisation: As shown in Section II-B, the complexe robot tends towards the goal during the optimisation process. At early stages, i.e. when the robot starts, having the full solution with all footsteps and final pose is not necessary. In fact, even a full solution is found, it is highly likely that this solution will change during walking motion to compensate sensory errors. The planner can keep up with the controller by only replan, at each correction, to an intermediate state. Namely, line 14 in Algorithm 2 will be modified to: "replan to new solution or timeout", where the timeout chosen is one stepping period.

While stop and go strategy guarantees optimised trajectory at each replanning stage, it produces unnatural movements on the robot. Using interative approach, the robot does not have to stop while correcting its paths towards the new goal. However, this method relies on the assumption that the intermediate

\begin{tabular}{c|r|r|r} 
Goal modification $(\mathrm{m})$ & Max $(\mathrm{ms})$ & $\operatorname{Min}(\mathrm{ms})$ & Average $(\mathrm{ms})$ \\
\hline 0.01 & 16 & 8 & 10.7 \\
0.02 & 38 & 7 & 11.4 \\
0.05 & 12 & 9 & 11.0 \\
0.1 & 48 & 9 & 15.5 \\
0.2 & 23 & 11 & 20.0 \\
0.5 & 117 & 15 & 33.6
\end{tabular}

TABLE II

CPU TIME FOR REPLANNING FOOTSTEPS (MILISECONDS) FOR A LOCOMOTION-GRASP TASK FOR A LOCOMOTION-GRASP TASK (FIGURE 1)

stage found at each timeout is safe for the robot.

As it turns out, there exists a third strategy which consists in reducing the dimension of the optimisation problem in replanning stage by blocking some or all joints belonging to the upper body. At each replanning step, optimised foot placements and posture are found without compromising the time constraint. Without having to interupt the optimisation process at each timeout as the second approach, this method is also simpler to implement on the robot. This thrird strategy is chosen for experiement and is detailed in the following section.

\section{B. Realtime footstep replanning}

While footsteps have to be changed to correct execution drift or perception error, the posture does not. In fact, unless new obstacles are found near the goal the previous posture stays valid. For example, if the robot is to approach and open a door, even the position of the door on its reference has change, the previous posture still guarantees dynamic equilibium. This scenario covers most cases in experiment. The most part of the last $n$ degrees of freedom of the the augmented robot can be 'frozen' while other joints are free to move. Suppose $l$ degrees of freedom are locked. This translates into reducing the dimension of the Jacobians, hence the complexity of the problem.

Table II shows replanning time in a grasping scenario similar to the one described in previous section with the posture of the standing robot "frozen". This is the other extreme case compared to Table I where every joint in the standing robot is free to move. In the first replanning scheme, the robot can barely correct small errors, so the robot has to make many small corrections at a time, hence a large amounts of supplement steps have to be added to the locomotion stage. In the second replanning schema however, the replan time is much less than time necessary for the robot to make a step. This guarantees a realtime replanning running behind the scene and updating robot footprints continuously.

Extension to footstep replanning of an arbitrary initial path: In cases where all upper-body joints are blocked in the replanning phase, this method can be extended to a variety of motion. In fact, the inputs for the planner is just initial footsteps and postures, which can be given by a different planner than the one described in section II-B, for example, an RRT planner. The robot can therefore deals with more complexe cases, typically scenarios where the optimisation method fails due to local minima, for example, a long wall is placed between the robot and goal. 


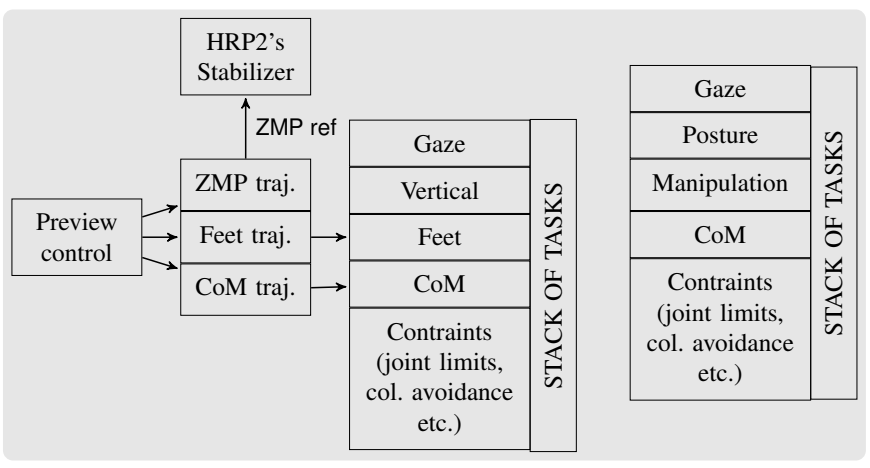

Fig. 7. Stack of task during walking (left) and manipulation (right)

\section{Control}

With the plan (final posture and footsteps) being updated in realtime, it is up to the controller to executed the plan. In this framework the "stack-of-tasks" [18]-[20] is used. The principle of task-based control is similar to the formulation of optimisation problem in Section II-B.

In this framework the control law $\dot{\boldsymbol{q}}_{\mathbf{i}}$ is written as following:

$$
\dot{\mathbf{q}}_{\mathbf{i}}=-\lambda J_{i}^{+} \mathbf{e}_{\mathbf{i}}
$$

$J_{i}^{+}$is the pseudo-inverse of the Jacobian $J_{i}$ [9]. $\mathbf{e}_{\mathbf{i}}$ is the difference between desired feature $\mathbf{s}_{\mathbf{i}}^{*}$ (i.e. a position in operational space, a posture, etc. ) and its current value $\mathbf{s}_{\mathbf{i}}$ :

$$
\mathbf{e}_{\mathbf{i}}=\mathbf{s}_{\mathbf{i}}-\mathbf{s}_{\mathbf{i}}^{*}
$$

The control law on a set of tasks is written [21]:

$$
\dot{\mathbf{q}}_{i}=\dot{\mathbf{q}}_{i-1}+\lambda_{i} \bar{J}_{i}^{+}\left(\dot{\mathbf{e}}_{i}-J_{i} \dot{\mathbf{q}}_{i-1}\right), \quad \dot{\mathbf{q}}_{1}=\lambda_{1} \bar{J}_{1}^{+} \dot{\mathbf{e}}_{1}
$$

when $\bar{J}_{i}$ is the projection of $J_{i}$ in the null space of the augmented Jacobian

$$
\begin{gathered}
J_{i}^{A}=\left[J_{1}, J_{2}, \ldots J_{i-1}\right]^{T} \\
\bar{J}_{i}=J_{i} P_{i-1}^{A}, \quad P_{i}^{A}=I-\left(J_{i}^{A}\right)^{+} J_{i}^{A}
\end{gathered}
$$

$\bar{J}_{1}^{+}$is simply $J_{1}^{+}$. One recovers (9) if there is one task in the stack. This formulation guarantees that the task at ith stage does not disturb the previous tasks, i.e. with higher priority. It allows, for instance, to realize a reaching movement without moving the center of mass, which is required for stability.

1) Application: In the controller perspective, there is no difference between walking and manipulating an object. Each of those stages is just a collection of tasks. Figure 7. depicts the corresponding stack in the two cases.

a) Walking: To ensure the dynamic equilibrium of the robot during walking, a preview control on the Zero Moment Point (ZMP) is used. The principle of this approach is to use an inverted pendulum model [4], the mass of the pendulum being that of the robot positioned at the height of the center of mass. The dynamic walking of a humanoid robot is

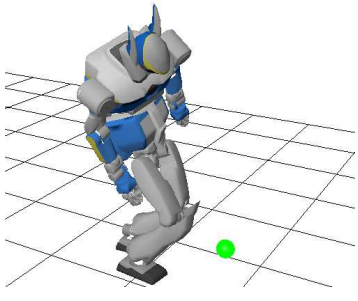

Fig. 8. Blocking position due to reaching task

modeled by a moving cart model [5], [6] [22]. The inputs of the preview-controller are the footprints to be realized. The outputs are the trajectories of the feet, center of mass, and the ZMP. These trajectories are injected into the StackOfTasks as position and orientation tasks on corresponding operational points. Obviously, self-collision avoidance tasks are put into the stack with the highest priority, next comes the task of monitoring trajectories (feet, CoM) in the final task was the vertically of the upper body.

b) Upper body manipulation: Object manipulation is done in a similar manner. In fact, using the stack-of-tasks manipulation and walking are merged in the same framework. Stability in the manipulation phase is ensured by the fact that the task of the center of mass is at highest priority, just like in the locomotion phase.

One last issue is the situations depicted in Figure 8. In these cases, simply adding the grasping task at the same time as posture task, the controller might try to get the hand the fastest possible to the goal and the hand gets stucked behind the body. The remedy here is activating the posture task first, then using a simple heuristic to activate hand task only after sometime at an appropriate time. In practice, placing a threshold on the hand task error and activating the hand task accordingly provides good results.

\section{EXPERIMENTS}

Described here are some scenarios with the humanoid robot HRP-2 using the proposed method.

\section{A. Reach and open a door}

In this scenario, (Figure 9) the robot has to walk towards a door, reaching for the handle and pull to open the door by walking backwards.

\section{B. Catching a flying balloon}

The robot must follow and catch a falling balloon. (Figure 10). No assumption is made about the dynamics of the balloon. The robot simply try to place its hands at certain height at the right position to catch the ball. In simulation, a user alters the wind condition to change the trajectory of the balloon hand changing robot planned motion online.

\section{Grasping a moving ball on the ground at distance}

This example illustrates probably the best the connection between locomotion and manipulation since precise conditions have to be met for the robot footsteps to make the grasping 


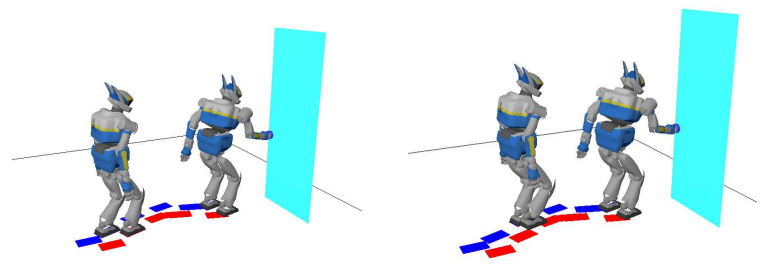

Fig. 9. Reach and open a door

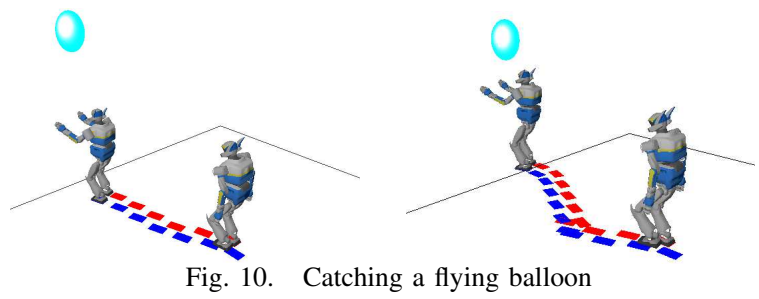

dynamically stable. Similar to the catching scenario, the position of the ball changes during walking and the robot must adapt to this change (Figure 1).

\section{CONCLUSION REMARKS AND FUTURE WORKS}

A generic method for footstep and posture planning and real time replanning has been presented in this paper. Numerous scenarii have demonstrated the applicability of this framework. All demonstrated motion have been tested dynamically with the simulator OpenHRP. On the real robot, the goal dectection and tracking using using CAMShift [23] algorithm acts as the perception module. Experiments have been conducted on the robot with HRP-2 following and reaching a ball on the ground, with the goal eventually moved during experiment.

Expansion can be made to this scheme of footsteps replanning in more dynamically intensive cases, e.g catching a volleyball instead of a balloon. In this cases, more precise model for the ZMP will have to be investigated to make fast steps possible. Modifications will also need to be done in the feasibility constraints of the virtual kinematic chain.

\section{REFERENCES}

[1] T. Sugihara, Y. Nakamura, and H. Inoue, "Real-time humanoid motion generation through zmp manipulation based on inverted pendulum control", in Robotics and Automation, 2002. Proceedings. ICRA 'O2. IEEE International Conference on, 2002, vol. 2, pp. 1404 - 1409 vol.2.

[2] K. Nishiwaki, S. Kagami, Y. Kuniyoshi, M. Inaba, and H. Inoue, "Online generation of humanoid walking motion based on a fast generation method of motion pattern that follows desired zmp", in Intelligent Robots and Systems, 2002. IEEE/RSJ International Conference on, 2002, vol. 3, pp. $2684-2689$ vol.3.

[3] K. Nishiwaki and S. Kagami, "High frequency walking pattern generation based on preview control of zmp", in Robotics and Automation, 2006. ICRA 2006. Proceedings 2006 IEEE International Conference on, may 2006, pp. $2667-2672$.

[4] S. Kajita, F. Kanehiro, K. Kaneko, K. Yokoi, and H. Hirukawa, "The 3d linear inverted pendulum mode: a simple modeling for a biped walking pattern generation", in Intelligent Robots and Systems, 2001. Proceedings. 2001 IEEE/RSJ International Conference on, 2001, vol. 1, pp. $239-246$ vol.1.

[5] S. Kajita, F. Kanehiro, K. Kaneko, K. Fujiwara, K. Harada, K. Yokoi, and $\mathrm{H}$. Hirukawa, "Biped walking pattern generation by using preview control of zero-moment point", in Robotics and Automation, 2003. Proceedings. ICRA '03. IEEE International Conference on, 2003, vol. 2, pp. $1620-1626$ vol.2.
[6] S. Kajita, M. Morisawa, K. Harada, K. Kaneko, F. Kanehiro, K. Fujiwara, and H. Hirukawa, "Biped walking pattern generator allowing auxiliary zmp control", in Intelligent Robots and Systems, 2006 IEEE/RSJ International Conference on, 2006, pp. 2993 -2999.

[7] Satoshi Kagami, Tomonobu Kitagawa, Koichi Nishiwaki, Tomomichi Sugihara, Masayuki Inaba, and Hirochika Inoue, "A fast dynamically equilibrated walking trajectory generation method of humanoid robot", Autonomous Robots, vol. 12, pp. 71-82, 2002, 10.1023/A:1013210909840.

[8] Yoshihiko Nakamura, Hideo Hanafusa, and Tsuneo Yoshikawa, "Taskpriority based redundancy control of robot manipulators", The International Journal of Robotics Research, vol. 6, no. 2, pp. 3-15, 1987.

[9] O. Khatib, "A unified approach for motion and force control of robot manipulators: The operational space formulation", Robotics and Automation, IEEE Journal of, vol. 3, no. 1, pp. 43 -53, 1987.

[10] C. Ott, O. Eiberger, W. Friedl, B. Bauml, U. Hillenbrand, C. Borst, A. Albu-Schaffer, B. Brunner, H. Hirschmuller, S. Kielhofer, R. Konietschke, M. Suppa, T. Wimbock, F. Zacharias, and G. Hirzinger, "A humanoid two-arm system for dexterous manipulation", in Humanoid Robots, 2006 6th IEEE-RAS International Conference on, dec. 2006, pp. $276-283$.

[11] L. Sentis and O. Khatib, "A whole-body control framework for humanoids operating in human environments", in Robotics and Automation, 2006. ICRA 2006. Proceedings 2006 IEEE International Conference on, May 2006, pp. $2641-2648$.

[12] M. Gienger, H. Janssen, and C. Goerick, "Task-oriented whole body motion for humanoid robots", in Humanoid Robots, 2005 5th IEEE-RAS International Conference on, dec. 2005, pp. $238-244$.

[13] J. Chestnutt, M. Lau, G. Cheung, J. Kuffner, J. Hodgins, and T. Kanade, "Footstep planning for the honda asimo humanoid", in Robotics and Automation, 2005. ICRA 2005. Proceedings of the 2005 IEEE International Conference on, 2005, pp. 629 - 634.

[14] S. Kagami, K. Nishiwaki, Jr. Kuffner, J.J., Y. Kuniyoshi, M. Inaba, and $\mathrm{H}$. Inoue, "Online $3 \mathrm{~d}$ vision, motion planning and bipedal locomotion control coupling system of humanoid robot: H7", in Intelligent Robots and Systems, 2002. IEEE/RSJ International Conference on, 2002, vol. 3, pp. $2557-2562$ vol.3.

[15] N. Perrin, O. Stasse, F. Lamiraux, P. Evrard, and A. Kheddar, "On the problem of online footsteps correction for humanoid robots", in The 27th annual Conference of the Robotics Society of Japan, 2009, September 15-17, Yokohama, Japan.

[16] Oussama Kanoun, Florent Lamiraux, Pierre-Brice Wieber, Fumio Kanehiro, Eiichi Yoshida, and Jean-Paul Laumond, "Prioritizing linear equality and inequality systems: application to local motion planning for redundant robots", in Proceedings of the 2009 IEEE international conference on Robotics and Automation, Piscataway, NJ, USA, 2009. ICRA'09, pp. 724-729, IEEE Press.

[17] Oussama Kanoun, Jean-Paul Laumond, and Eiichi Yoshida, "Planning foot placements for a humanoid robot: A problem of inverse kinematics", The International Journal of Robotics Research, 2010.

[18] N. Mansard and F. Chaumette, "Task sequencing for high-level sensorbased control", Robotics, IEEE Transactions on, vol. 23, no. 1, pp. 60 $-72,2007$.

[19] N. Mansard, O. Khatib, and A. Kheddar, "A unified approach to integrate unilateral constraints in the stack of tasks", Robotics, IEEE Transactions on, vol. 25, no. 3, pp. $670-685,2009$.

[20] O. Stasse, A. Escande, N. Mansard, S. Miossec, P. Evrard, and A. Kheddar, "Real-time (self)-collision avoidance task on a hrp-2 humanoid robot", in Robotics and Automation, 2008. ICRA 2008. IEEE International Conference on, May 2008, pp. 3200 -3205.

[21] B. Siciliano and J.-J.E. Slotine, "A general framework for managing multiple tasks in highly redundant robotic systems", in Advanced Robotics, 1991. 'Robots in Unstructured Environments', 91 ICAR., Fifth International Conference on, June 1991, pp. 1211 -1216 vol.2.

[22] M. Morisawa, S. Kajita, K. Kaneko, K. Harada, F. Kanehiro, K. Fujiwara, and H. Hirukawa, "Pattern generation of biped walking constrained on parametric surface", in Robotics and Automation, 2005. ICRA 2005. Proceedings of the 2005 IEEE International Conference on, 2005, pp. $2405-2410$.

[23] G. R. Bradski, "Conputer vision face tracking for use in a perceptual user interface", Proc. of Intel Technology Journal, 1998, 1998. 